\title{
Spectrum Index: IQ deviations, rural disparities and underweight infants
}

\author{
BY NIKO MCCARTY
}

23 FEBRUARY 2022

Welcome to Spectrum Index, a monthly newsletter that distills data from noteworthy autism studies into a series of quick statistics. Let us know what you think at news@spectrumnews.org, and thanks for reading.

25.75: The average standard deviation in intelligence quotient (IQ) for 671 autistic children, aged 6 through 16 . Children with autism are 1.5 times as likely as their non-autistic peers to have a 'superior' IQ, defined as a score of 120 or higher, and 12 times as likely to have intellectual disability. The results were published in Child Psychiatry \& Human Development in January.

1.28: The fold increase in likelihood that a child from an urban or suburban area will be diagnosed with autism, compared with a child in a rural area, according to a study published February in the Journal of Autism and Developmental Disorders. The finding, based on more than 176,000 babies born in Taiwan in 2006, somewhat contradicts prior work suggesting that universal health care had largely eliminated disparities between urban and rural preventive care and checkups in Taiwan.

3.25: The fold increase in the odds that an underweight infant, weighing 1.5 to 1.9 kilograms (3.3 to 4.2 pounds) at birth, will later be diagnosed with autism, compared with an infant who weighs more than 2.5 kilograms, or 5.5 pounds. The study, published in Scientific Reports in February, drew upon nearly 2.2 million insurance records in Korea. Infants who weighed 2 to 2.4 kilograms (4.4 to 5.3 pounds) also had a 1.91 times increased odds of a subsequent autism diagnosis.

74: The percentage of autistic children, out of more than 25,000 aged 4 to 8 , who reported having a sensory feature, such as an extreme sensitivity to sound or reduced feelings of pain. Published in Autism Research in January, the study also found that white children were most likely, compared with other races, to report such a condition. 


\section{Spectrum | Autism Research News}

https://www.spectrumnews.org

23: The increased chance, as a percentage, that someone with a childhood infection will later be diagnosed with autism. The heightened value is based on health records from more than 550,000 people living in Stockholm, Sweden, and emerged after controlling for sex, maternal body mass index, parental ages and education, family income and a slew of other potentially confounding factors. The work was published in the Journal of Neurodevelopmental Disorders in February.

Negative: The association between an autistic child's performance on the lowa Gambling Task a card-based decision-making test — and her outcome from cognitive behavioral therapy (CBT) aimed at reducing anxiety. The better children did on the gambling task, in other words, the fewer benefits they gained from standard CBT. The researchers did not see this association with a version of CBT adapted for children with autism, suggesting that autistic children can benefit more from the adapted form. The study, based on 148 autistic children aged 7 to 13 who had a score of 14 or higher on the Pediatric Anxiety Rating Scale, was published in the Journal of Clinical Child \& Adolescent Psychology in January.

Cite this article: https://doi.org/10.53053/KPZE4428 